\title{
TRACE RINGS OF GENERIC MATRICES ARE COHEN-MACAULAY
}

\author{
MICHEL VAN DEN BERGH
}

\section{INTRODUCTION}

Let $G$ be a reductive algebraic group over an algebraically closed field of characteristic zero, and let $W$ be a finite-dimensional representation of $G$. Then $G$ acts on the polynomial ring $k[W]$, and the Hochster-Roberts Theorem [8] tells us that $k[W]^{G}$ is Cohen-Macaulay.

A primary objective in this paper will be to study a situation that looks very similar. Let $U$ be another finite-dimensional $G$-representation. Then $U \otimes_{k}$ $k[W]$ is a free $k[W]$-module, and a natural generalization of the HochsterRoberts Theorem would be that $\left(U \otimes_{k} k[W]\right)^{G}$ is a Cohen-Macaulay $k[W]^{G}$ module.

Unfortunately, it is easy to see that this cannot be true in general (see Example 3.1). There is, however, a conjecture due to Stanley [20] that gives at least some cases under which the above statement is true.

We will not say anything about Stanley's original conjecture. Instead, we will replace it with a slightly weaker version (Conjecture $3.4^{\prime}$ ).

The first main result in this paper is that we prove Conjecture $3.4^{\prime}$ for certain pairs $(G, W)$. Namely, if $X=\operatorname{Spec} k[W]$ and $X^{u}$ is the locus of the $G$ unstable points in $X$, then we require that $X^{u}$ be constructible, i.e., that it can be built up from smaller manageable parts in a sense explained in $\S 6$. As a corollary, we immediately obtain that Conjecture $3.4^{\prime}$ holds if $G=S L_{2}$ (Theorem 6.1.11).

Another situation that can be handled by the methods developed in this paper is

$$
G=S L(V) \text { and } \quad W=\operatorname{End}(V)^{m *} .
$$

In the last section of this paper, we will show that in this case $X^{u}$ is constructible and hence Conjecture $3.4^{\prime}$ holds.

Our main motivation for studying (1) lies in our interest in the trace rings of generic matrices. Let $M_{n}$ be the variety of $n \times n$-matrices. $\left(M_{n}\right)^{m}$ will be the $m$-fold product (over Speck) $M_{n} \times M_{n} \times \cdots \times M_{n}$. Let $G=S L_{n}$. Then we define

$$
\mathbf{T}_{m, n}=\left\{f:\left(M_{n}\right)^{m} \rightarrow M_{n} \mid f \text { polynomial and } G \text {-equivariant }\right\} .
$$

Received by the editors March 3, 1989.

1980 Mathematics Subject Classification (1985 Revision). Primary 20G05, 16A38.

The author was supported by an NFWO grant. 
$\mathbf{T}_{m, n}$ is a noncommutative ring (using the multiplication in $M_{n}$ ), and its center is given by

$$
Z_{m, n}=\left\{f:\left(M_{n}\right)^{m} \rightarrow \operatorname{Spec} k \mid f \text { polynomial and } G \text {-equivariant }\right\} .
$$

$Z_{m, n}$ is the commutative and $\mathbf{T}_{m, n}$ is the noncommutative trace ring of $m$ generic $n \times n$-matrices. They were first extensively studied by M. Artin and C. Procesi. Artin and Schelter proved that the maximal ideals of $Z_{m, n}$ parametrize semisimple representations of dimension $n$ of the free algebra $k\left\langle X_{1}, \ldots, X_{m}\right\rangle$, and the two-sided maximal ideals of $\mathbf{T}_{m, n}$ correspond to the simple components of such representations $[1,2]$.

Let $X_{i}$ be the projection of $\left(M_{n}\right)^{m}$ onto the $i$ th factor, and let

$$
\operatorname{Tr}\left(X_{i_{1}} \cdots X_{i_{u}}\right):\left(M_{n}\right)^{m} \rightarrow \text { Speck }
$$

be the corresponding trace maps. Procesi proved Artin's conjecture that $Z_{m, n}$ is generated over $k$ by the trace monomials $\operatorname{Tr}\left(X_{i_{1}} \cdots X_{i_{u}}\right)$ and $\mathbf{T}_{m, n}$ is generated as a module over $Z_{m, n}$ by the monomials in the $X_{i}^{u}$ 's [16]. Furthermore, he proved that all the relations between those generators can be obtained from the Cayley-Hamilton polynomial (explaining the terminology of trace rings).

From this, we easily deduce that $\mathbf{T}_{m, n}$ is a generic object in the category of $k$-algebras with a trace map. To be more precise, let $\Lambda$ be a $k$-algebra, equipped with a further unary operation $T: \Lambda \rightarrow \Lambda$, called trace, satisfying the list of conditions in [17, p. 194]. Assume, furthermore, that $T$ satisfies the Cayley-Hamilton identities of $n \times n$-matrices. Let $a_{1}, \ldots, a_{m} \in \Lambda$. Then there exists a unique map $\mathbf{T}_{m, n} \rightarrow \Lambda$ commuting with trace and sending $X_{i}$ to $a_{i}$.

If $n=1$, then $\mathbf{T}_{m, n}$ is a polynomial ring, and hence it has finite global dimension. A first natural question would be whether $\mathbf{T}_{m, n}$ always has finite global dimension (being a generic object). However, the complete, somewhat disappointing result is given below.

Theorem 1.1 $[5,11,12,18] . \mathbf{T}_{m, n}$ has finite global dimension if and only if $n=1, m=1,(m, n)=(2,2),(m, n)=(3,2)$, or $(m, n)=(2,3)$.

After computations in low dimensions, $\mathbf{L}$. le Bruyn conjectured that $\mathbf{T}_{m, n}$ is always a Cohen-Macaulay $Z_{m, n}$-module. This was proved by him in the case of $2 \times 2$-matrices. Later, he and Procesi proved that $\left(\mathbf{T}_{m, n}\right)_{p}$ is Cohen-Macaulay if $p \in \operatorname{Max} Z_{m, n}$ corresponds to a semisimple representation of $k\left\langle X_{1}, \ldots, X_{m}\right\rangle$ having distinct irreducible components [11].

As the title of this paper indicates, we will prove that $\mathbf{T}_{m, n}$ is CohenMacaulay in general (Theorem 7.3.6). This is done as follows. From (2), it is clear that

$$
\mathbf{T}_{m, n}=(U \otimes k[W])^{G} \text { where } G=S L(V), W=\operatorname{End}(V)^{m *}, U=\operatorname{End}(V) .
$$

Hence, we are in the situation of (1), and Conjecture $3.4^{\prime}$ applies. Since it is easy to see that the hypotheses of Conjecture $3.4^{\prime}$ are satisfied in this case, we are done. 
This paper is organized as follows.

In $\S 2$, we introduce some often used notation.

In $\S 3$, we review Stanley's conjecture, and we introduce the weaker version, Conjecture $3.4^{\prime}$.

In $\S 4$, we outline our method for verifying the Cohen-Macaulayness of $(U \otimes k[W])^{G}$. I.e., we relate this problem to the cohomology modules $H_{X^{u}}\left(X, \mathscr{O}_{X}\right)$.

In $\S 5$, we introduce a spectral sequence (Theorem 5.1) that may be interesting in its own right. It allows us, in some cases, to estimate the cohomology modules introduced above.

In $\S 6$, we try to break up $X^{u}$ into manageable pieces that can be handled by the main result of $\S 5$. This leads us to the notion of constructibility. We prove Conjecture $3.4^{\prime}$ for $G=S L_{2}$ (Theorem 6.1.11). In the last subsection, we introduce a combinatorial method for verifying constructibility.

Finally, in $\S 7$, we use the combinatorial criterion derived in $\S 6$ to show that $X^{u}$ is constructible in (1). We obtain that $\mathbf{T}_{m, n}$ is Cohen-Macaulay in general (Theorem 7.3.6).

\section{NOTATION AND CONVENTIONS}

In the sequel, $k$ will always be an algebraically closed field of characteristic zero.

If $G$ is a linear algebraic group over $k$, then $\mathscr{W}_{G}$ will be the Weyl group of $G$. $Y(G)$ will be the pointed set of one-parameter subgroups of $G$. An irreducible representation of $G$ defines a character $G \rightarrow G_{m}$. This is a polynomial map, invariant under conjugation (we will always assume that characters are characters of irreducible representations). If $T$ is a torus, then the characters of $T$ are homomorphisms, and they form an abelian group in the usual way. This group will be denoted by $X(T)$, and the group law will be written additively. We define $X(T)_{\mathbf{Q}}$ as $\mathbf{Q} \otimes_{\mathbf{Z}} X(T)$. Since $T$ is a torus, $Y(T)$ also carries an abelian group structure, and there is a natural pairing $Y(T) \times X(T) \rightarrow X\left(G_{m}\right) \cong \mathbf{Z}$ given by composition. This pairing will be denoted by $\langle$,$\rangle .$

Characters of $T$ will be identified with one-dimensional representations of $T$. Hence the notation $\chi_{1} \oplus \chi_{2}$ for $\chi_{1,2} \in X(T)$ stands for the two-dimensional representation of $T$ which is the direct sum of the one-dimensional representations determined by $\chi_{1}$ and $\chi_{2}$. This is not to be confused with $\chi_{1}+\chi_{2}$, which is just the sum of $\chi_{1}$ and $\chi_{2}$ in $X(T)$.

If $P \subset G$ is an algebraic subgroup of $G$ and $X$ is a scheme with a $P$-action, then $G \times{ }^{P} X=G \times X / P$. There is a natural projection map $G \times{ }^{P} X \rightarrow G / P$ given by $\overline{(g, x)} \mapsto \bar{g}$ with fibers isomorphic to $X$. Taking the fiber over $[P]$ in $G / P$ induces an equivalence between the category of quasicoherent $\mathscr{O}_{G \times{ }^{P} X^{-}}$ modules with a $G$-action and the category of quasicoherent $\mathscr{O}_{X}$-modules with a $P$-action. The inverse of this equivalence will be denote by $\sim$

Let $R$ be a $\mathbf{Z}$-graded Noetherian commutative ring of the form $k \oplus R_{1} \oplus$ $R_{2} \oplus \cdots$, and let $M$ be a finitely generated graded $R$-module. The Poincaré 
series of $M$ will be defined as

$$
P(M, t)=\sum_{n=-\infty}^{+\infty} \operatorname{dim}\left(M_{n}\right) t^{n} .
$$

When we say that $M$ is Cohen-Macaulay, we always mean that $M$ is maximal Cohen-Macaulay. This is equivalent to the fact that $R$ contains a graded polynomial ring $R^{\prime}$ over $k$ such that $M$ is a finitely generated free $R^{\prime}$-module.

\section{A conjecture of Stanley}

In this section, we will disucss a natural generalisation for the HochsterRoberts Theorem on Cohen-Macaulayness of invariant rings. Unfortunately, this generalisation is not true in general. There exists, however, a conjecture due to Stanley [20] which gives at least some cases under which the generalisation is true.

Let $G$ be a reductive group over $k$, and let $U, W$ be two finite-dimensional representations of $G$. Define $R=k[W], d=\operatorname{dim} W$, and $h=\operatorname{dim} R^{G}$. Then $G$ acts in a natural way on the free $R$-module $U \otimes_{k} R$.

By the Hochster-Roberts Theorem [8], $R^{G}$ is Cohen-Macaulay. It is, therefore, natural to ask whether $\left(U \otimes_{k} R\right)^{G}$ is a Cohen-Macaulay $R^{G}$-module. This is not always true. Here is a simple counterexample.

Example 3.1. Let $G=T=G_{m}$, and let $\chi$ be a generator for $X(T)$. Define $U=\chi^{-1}$ and $W=\chi \oplus \chi \oplus \chi^{-1}$. Then $R=k[x, y, z], M=k[x, y, z]$, and $G_{m}$ acts on $R$ and $M$ as follows: let $\alpha \in G_{m}, f \in R$, and $g \in M$. Then $\alpha \cdot f=f\left(\alpha x, \alpha y, \alpha^{-1} z\right)$ and $\alpha \cdot g=\alpha^{-1} g\left(\alpha x, \alpha y, \alpha^{-1} z\right)$. Hence, $R^{G}=$ $k[x z, y z]$ and $M^{G}=(x z, y z) z^{-1}$. Clearly, $M^{G}$ is not a Cohen-Macaulay $R^{G}$-module.

It is no restriction to assume that $U$ is irreducible because if $U=U_{1} \oplus \cdots \oplus$ $U_{u}$, then clearly $(U \otimes R)^{G}=\left(U_{1} \otimes R\right) \oplus \cdots \oplus\left(U_{u} \otimes R\right)^{G}$. Hence, from now on we will make this assumption. In that case, $U^{*}$ is determined by its character $\chi: G \rightarrow G_{m}$.

For an arbitrary character of $G$, Stanley defines $R_{\chi}^{G}$ as the sum of all irreducible subrepresentations with character $\chi$ [20]. Clearly, $R=\oplus_{\chi} R_{\chi}^{G}$, where $\chi$ runs through all characters of $G$. The proof that $R^{G}$ is finitely generated also works for $R_{\chi}^{G}$, and since $R_{\chi}^{G}$ is obviously torsion free, we deduce that $\operatorname{dim} R_{\chi}^{G}=\operatorname{dim} R^{G}$ if $R_{\chi}^{G} \neq 0$.

Lemma 3.2. If $\chi$ is the character of $U^{*}$, then $R_{\chi}^{G} \cong U^{*} \otimes(U \otimes R)^{G}$.

Hence, the question whether $(U \otimes R)^{G}$ is Cohen-Macaulay is equivalent to the question whether $R_{\chi}^{G}$ is Cohen-Macaulay.

Assume now that $G=T$ is a torus, $\chi \in X(T)$, and let the weights of $W$ be given by $\alpha_{1}, \ldots, \alpha_{d} \in X(T)$. Then we say that $\chi$ is critical [20] for $(T, W)$ if 
the system $z_{1} \alpha_{1}+\cdots+z_{d} \alpha_{d}=\chi$ in $X(T)_{\mathbf{Q}}$ has a rational solution $\left(a_{1}, \ldots, a_{d}\right)$ with the following properties.

(1) $a_{i} \leq 0$.

(2) If $\left(b_{1}, \ldots, b_{d}\right)$ is an integer solution of $z_{1} \alpha_{1}+\cdots+z_{d} \alpha_{d}=\chi$ such that $b_{i} \geq a_{i}$, then $b_{i} \geq 0$ for all $i$.

Theorem 3.3 [20]. Assume that $\chi$ is critical for $(T, W)$. Then $R_{\chi}^{T}$ is CohenMacaulay. Furthermore, there is a functional equation

$$
P\left(R_{\chi}^{T}, 1 / t\right)=(-1)^{h} t^{d} P\left(R_{\psi}^{T}, t\right),
$$

where $\psi=(\chi \operatorname{det} \chi)^{*}$. Here ${ }^{*}$ denotes the dual character, and $\operatorname{det} \chi$ is the character of the highest exterior power of the representation corresponding to $\chi$.

A character is clearly critical if it is of the form $\sum_{i=1}^{d} a_{i} \alpha_{i}$, where $-1<a_{i} \leq$ 0 . We will call such a character strongly critical. This notion is useful because it is somewhat easier to check that a character is strongly critical than that it is critical.

Assume now that $G$ is arbitrary again, and let $T \subset G$ be a maximal torus. Assume that $\chi: G \rightarrow G_{m}$ is a character. Then $\chi \mid T=\chi_{1} \oplus \cdots \oplus \chi_{u}$, where $\chi_{i} \in X(T)$. Let $\left(\rho_{1}, \ldots, \rho_{r}\right)$ be the set of roots of $G$. Then Stanley [20] calls $\chi$ critical for $(G, W)$ if $\chi_{i}-\sum_{j \in S} \rho_{j}$ is critical for $(T, W)$ for all $1 \leq i \leq u$ and for all $S \subset\{1, \ldots, r\}$. He proves that if $\chi$ is critical, then $R_{\chi}^{G}$ satisfies the functional equation (4). This leads to a natural conjecture.

Conjecture 3.4. If $\chi$ is critical for $(G, W)$, then $R_{\chi}^{G}$ is Cohen-Macaualy.

Of course, a weaker version of this conjecture can be obtained if we require that all the $\chi_{i}-\sum_{j \in S} \rho_{j}$ are strongly critical. A character with this property will be called strongly critical for $(G, W)$. In the sequel, we will refer to the weaker version of Conjecture 3.4 as Conjecture $3.4^{\prime}$.

\section{THE METHOD}

As in the previous section, $G$ will be a reductive algebraic group over $k$. $R=k[W]$ and $d=\operatorname{dim} R ; h=\operatorname{dim} R^{G} \cdot \chi$ will be some character of $G$. We define $I=R\left(R^{G}\right)^{+}$.

The following lemma will be basic in this paper.

Lemma 4.1. $H_{\left(R^{G}\right)^{+}}^{\cdot}\left(R_{\chi}^{G}\right)=\dot{H_{I}^{*}}(R)_{\chi}^{G}$. (Here $H_{I}^{\cdot}(R)_{\chi}^{G}$ has the obvious meaning. ) Proof. Let $f_{1}, \ldots, f_{u}$ be a set of generators for $\left(R^{G}\right)^{+}$. Then the $\left(f_{i}\right)_{i}$ are obviously also $R$ generators for $I$. Let $K^{*}\left(R, f_{1}, \ldots, f_{u}\right)$ be the complex

$$
0 \rightarrow \bigoplus_{i} R_{f_{i}} \rightarrow \bigoplus_{\substack{i, j \\ i<j}} R_{f_{i} f_{j}} \rightarrow \cdots \rightarrow R_{f_{1} \cdots f_{u}} \rightarrow 0
$$


with the standard boundary maps. Then $H_{I}^{\cdot}(R)_{\chi}^{G}=H^{*}\left(K^{\cdot}\left(R, f_{1}, \ldots, f_{u}\right)\right)_{\chi}^{G}$. But using the fact that $G$ is reductive, we easily deduce

$$
\begin{aligned}
H^{\cdot}\left(K^{\cdot}\left(R, f_{1}, \ldots, f_{u}\right)\right)_{\chi}^{G} & =H^{\cdot}\left(K^{\cdot}\left(R, f_{1}, \ldots, f_{u}\right)_{\chi}^{G}\right) \\
& =H^{\cdot}\left(K^{\cdot}\left(R_{\chi}^{G}, f_{1}, \ldots, f_{u}\right)\right) \\
& =H_{\left(R^{G}\right)^{+}}^{\cdot}\left(R_{\chi}^{G}\right) .
\end{aligned}
$$

Corollary 4.2. $R_{\chi}^{G}$ is Cohen-Macaulay if and only if $H_{I}^{i}(R)_{\chi}^{G}=0$ for $i=0, \ldots, h-1$.

Proof. This statement is vacuous and hence true if $R_{\chi}^{G}=0$. So assume that $R_{\chi}^{G} \neq 0$. It is well known that $R_{\chi}^{G}$ is Cohen-Macaulay if and only if $H_{\left(R^{G}\right)^{+}}^{i}\left(R_{\chi}^{G}\right)=0$ for $i=0, \ldots, h-1$. Then the result follows from Lemma 4.1.

Let $X=\operatorname{Spec} k[W]$, and let $T$ be a maximal torus in $G$. The radical of the ideal $I$ is the defining ideal of the $G$-unstable locus in $X$ which will be denoted by $X^{u}$. I.e.,

$$
X^{u}=\{x \in X \mid 0 \in \overline{G x}\} .
$$

$X^{u}$ may be described more conveniently using the Hilbert-Mumford criterion [14] which says that every point in $X^{u}$ is unstable for some one-parameter subgroup of $G$. I.e., if $\lambda \in Y(G)$, then we define

$$
X_{\lambda}=\left\{x \in X \mid \lim _{t \rightarrow 0} \lambda(t) x=0\right\}
$$

and

$$
G(\lambda)=\left\{g \in G \mid \lim _{t \rightarrow 0} \lambda(t) g \lambda(t)^{-1} \text { exists }\right\} .
$$

Then $G(\lambda)$ acts on $X_{\lambda}$, and $G(\lambda)$ is a parabolic subgroup of $G[14$, Proposition 2.6]. Then it follows from the Hilbert-Mumford criterion that

$$
X^{u}=\bigcup_{\lambda \in Y(T)} G X_{\lambda} .
$$

$G X_{\lambda}$ is the image of $G \times{ }^{G(\lambda)} X_{\lambda}$ in $X$ under the canonical map. This map factors through the projection map $G / G(\lambda) \times X \rightarrow X$, and hence it is projective. Therefore, its image is closed (this is a well-known argument, see for example [13]).

Clearly, $G X_{\lambda}=G X_{w(\lambda)}$ if $w \in \mathscr{W}_{G}$. Therefore, we can restrict the union in (5) to a Weyl chamber in $Y(T)$. Let $B$ be a Borel subgroup of $G$ containing $T$. Then we have proved the following (well-known) fact.

Lemma 4.3. With notation and assumptions as above,

$$
X^{u}=\bigcup_{\substack{\lambda \in Y(T) \\ G(\lambda) \supset B}} G X_{\lambda} .
$$




\section{A RESUlT ON COHOMOLOGY WITH SUPPORT}

In Lemma 3.2, we saw that to check Cohen-Macaulayness of modules of invariants, it is important to be able to compute cohomology with support in the unstable locus.

Our two main tools to handle this problem will be the standard long exact sequence for cohomology with support and Theorem 5.1 below.

Theorem 5.1. Assume that we are in the following situation:

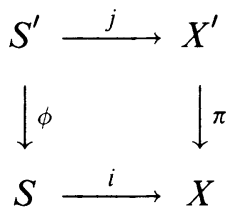

where

(1) $X, X^{\prime}$ are smooth over $k$, and $\pi$ is a smooth projective map.

(2) $i$ and $j$ are closed immersions.

(3) $\phi$ is the restriction of $\pi$ to $S^{\prime}$, and it is set-theoretically a bijection.

Let $\mathscr{M}$ be a quasicoherent sheaf on $X$. Then there is a spectral sequence

$$
E_{1}^{p q}: H_{S^{\prime}}^{q}\left(X^{\prime}, \pi^{*} \mathscr{M} \otimes \bigwedge^{p} \Omega_{X^{\prime} / X}\right) \Rightarrow H_{S}^{p+q-2 \operatorname{dim}\left(X^{\prime} / X\right)}(X, \mathscr{M}) .
$$

The rest of this section will be devoted to the proof of this theorem.

Lemma 5.2. Let $I, I^{\prime}$ be the defining ideals of $S, S^{\prime}$ in $X, X^{\prime}$. Define $K_{t, S, X}^{\cdot}$ as the complex of $\mathscr{O}_{X}$-modules

$$
\begin{aligned}
& \pi_{*}\left(\mathscr{O}_{X^{\prime}} / I^{\prime t}\right) \stackrel{d_{0}}{\longrightarrow} \pi_{*}\left(\mathscr{O}_{X^{\prime}} / I^{\prime t-1} \otimes_{\mathscr{O}_{X^{\prime}}} \Omega_{X^{\prime} / X}\right) \stackrel{d_{1}}{\longrightarrow} \\
& \quad \ldots \stackrel{d_{q-1}}{\longrightarrow} \pi_{*}\left(\mathscr{O}_{X^{\prime}} / I^{\prime t-q} \otimes_{\mathscr{O}_{X^{\prime}}} \bigwedge^{q} \Omega_{X^{\prime} / X}\right) \stackrel{d_{q}}{\longrightarrow} \cdots,
\end{aligned}
$$

where $d_{q}$ is obtained from the exterior differentiation

$$
d: \bigwedge^{q} \Omega_{X^{\prime} / X} \rightarrow \bigwedge^{q+1} \Omega_{X^{\prime} / X} .
$$

The canonical map $\mathscr{O}_{X} / I^{t} \stackrel{u}{\longrightarrow} \pi_{*}\left(\mathscr{O}_{X^{\prime}} / I^{\prime t}\right)$ defines a complex

$$
0 \rightarrow \mathscr{O}_{X} / I^{t} \stackrel{u}{\longrightarrow} K_{t, S, X}^{\cdot}
$$

Let $\mathscr{T}$ be a quasicoherent injective $\mathscr{O}_{X}$-module. Then the complex obtained by applying $\underset{t}{\lim } \operatorname{Hom}(-, \mathscr{T})$ to (8) is exact.

We will not prove this lemma directly. Instead, we will treat a special case first.

Lemma 5.3. If $\phi$ is an isomorphism and $S$ is smooth, then (8) is exact.

Proof. $\pi_{*}$ is exact on coherent modules with support in $S^{\prime}$. Hence, (8) may be filtered in such a way that the associated graded complexes are of the form

$$
0 \rightarrow I^{s-1} / I^{s} \stackrel{\bar{u}}{\longrightarrow} \pi_{*}\left(I^{\prime s-1} / I^{\prime s}\right) \stackrel{\bar{d}_{0}}{\longrightarrow} \pi_{*}\left(I^{\prime s-2} / I^{\prime s-1} \otimes \Omega_{X^{\prime} / X}\right) \stackrel{\bar{d}_{1}}{\longrightarrow} \cdots .
$$


Then for (8) to be exact, it is sufficient that (9) is exact for all $s$. Furthermore, the hypotheses imply that the sequences (9) are sequences of vector bundles on $\mathscr{O}_{S}$.

Since $S$ and $S^{\prime}$ are smooth, we know that $I^{s-1} / I^{S}=S^{S-1}\left(I / I^{2}\right)$ and $I^{\prime s-1} / I^{\prime s}=S^{s-1}\left(I^{\prime} / I^{\prime 2}\right)$. With these identifications, the differentiation $\bar{d}_{q}$ is given by (on an affine open set)

$$
\begin{aligned}
& \bar{d}_{q}\left(a_{1} S a_{2} \cdots S a_{s-q} \otimes d b_{1} \wedge \cdots \wedge d b_{q}\right) \\
& \quad=\sum_{i=1}^{s-q} a_{1} S \cdots S \hat{a}_{i} S \cdots S a_{s-q} \otimes d a_{i} \wedge d b_{1} \wedge \cdots \wedge d b_{q} .
\end{aligned}
$$

For $s=1$, the sequence (9) reads as

$$
0 \rightarrow \mathscr{O}_{S} \rightarrow \pi_{*} \mathscr{O}_{S^{\prime}} \rightarrow 0
$$

which is obviously exact.

For $s=2$, we obtain

$$
0 \rightarrow I / I^{2} \rightarrow \pi_{*}\left(I^{\prime} / I^{\prime 2}\right) \rightarrow \pi_{*}\left(\Omega_{X^{\prime} / X} \otimes \mathscr{O}_{S^{\prime}}\right) \rightarrow 0 .
$$

Exactness of this sequence is obtained from the following diagram:

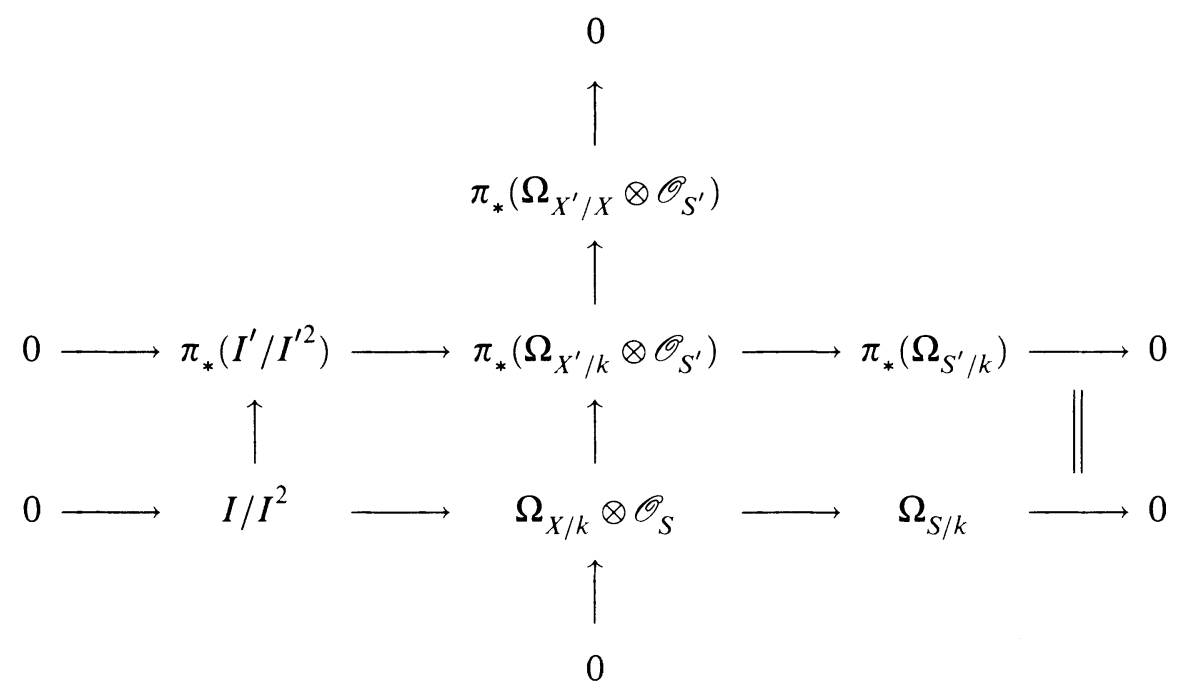

Here the vertical exact sequence is obtained by applying $\pi_{*}\left(-\otimes \mathscr{O}_{S^{\prime}}\right)$ to the standard exact sequence

$$
0 \rightarrow \pi^{*}\left(\Omega_{X / k}\right) \rightarrow \Omega_{X^{\prime} / k} \rightarrow \Omega_{X^{\prime} / X} \rightarrow 0
$$

for smooth maps.

Finally, for $s \geq 2$, we deduce from (10) that (9) is obtained from (11) by taking exterior powers. Hence, (9) is exact for $s \geq 2$.

Proof of Lemma 5.2. Our proof will be by induction on the dimension of $S$. We may clearly reduce to the case where $S$ and $S^{\prime}$ are reduced. In that case, there will be an open subvariety $S_{1}^{\prime}$ of $S^{\prime}$ such that $\phi \mid S_{1}^{\prime}$ is an isomorphism. 
By making $S_{1}^{\prime}$ smaller if necessary, we can also assume that $S_{1}^{\prime}$ is smooth. Define $S_{2}^{\prime}=S^{\prime} \backslash S_{1}^{\prime}, S_{1}=\phi\left(S_{1}^{\prime}\right), S_{2}=\phi\left(S_{2}^{\prime}\right), X_{1}=X \backslash S_{2}$, and $X_{1}^{\prime}=\pi^{-1}\left(X_{1}\right)$.

By our induction hypothesis and by Lemma 5.3, we may assume that Lemma 5.2 has been proved in the situations

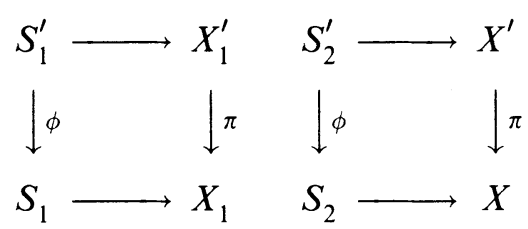

To complete our induction step, we need another lemma.

Lemma 5.4. Let $X$ be a Noetherian scheme, and let $U \subset X$ be an open subset. Let $I$ be the defining ideal of $X \backslash U$. Then for a quasicoherent injective $\mathscr{O}_{X^{-}}$ module $\mathscr{T}$ and a coherent $\mathscr{O}_{X}$-module $\mathscr{M}$, the following sequence is exact.

$$
\begin{aligned}
0 & \rightarrow \underset{s}{\lim } \operatorname{Hom}_{\mathscr{O}_{X}}\left(\mathscr{M} / I^{s} \mathscr{M}, \mathscr{T}\right) \rightarrow \operatorname{Hom}_{\mathscr{O}_{X}}(\mathscr{M}, \mathscr{T}) \\
& \rightarrow \operatorname{Hom}_{\mathscr{O}_{U}}(\mathscr{M}|U, \mathscr{T}| U) \rightarrow 0,
\end{aligned}
$$

where the maps are the obvious ones.

Proof. Well known and easy.

Now let $I_{2}, I_{2}^{\prime}$ be the defining ideals of $S_{2}, S_{2}^{\prime}$ in $X, X^{\prime}$. From the lemma, we obtain exact sequences

$$
\begin{aligned}
0 & \rightarrow \underset{t}{\lim } \underset{s}{\lim } \operatorname{Hom}_{\mathscr{O}_{X}}\left(\pi_{*}\left(\mathscr{O}_{X^{\prime}} / I^{\prime t} \otimes \bigwedge^{q} \Omega_{X^{\prime} / X}\right) \otimes \mathscr{O}_{X} / I_{2}^{s}, \mathscr{T}\right) \\
& \rightarrow \underset{t}{\lim } \operatorname{Hom}_{\mathscr{O}_{X}}\left(\pi_{*}\left(\mathscr{O}_{X^{\prime}} / I^{\prime t} \otimes \bigwedge^{q} \Omega_{X^{\prime} / X}\right), \mathscr{T}\right) \\
& \rightarrow \underset{t}{\lim } \operatorname{Hom}_{\mathscr{O}_{X_{1}}}\left(\pi_{*}\left(\mathscr{O}_{X^{\prime}} / I^{\prime t} \otimes \bigwedge^{q} \Omega_{X^{\prime} / X}\right)\left|X_{1}, \mathscr{T}\right| X_{1}\right) \rightarrow 0 .
\end{aligned}
$$

But by a standard argument,

$$
\begin{aligned}
\underset{t}{\lim } \underset{s}{\lim } \operatorname{Hom}_{\mathscr{O}_{X}}\left(\pi_{*}\left(\mathscr{O}_{X^{\prime}} / I^{\prime t} \otimes \bigwedge^{q} \Omega_{X^{\prime} / X}\right) \otimes \mathscr{O}_{X} / I_{2}^{s}, \mathscr{T}\right) \\
\quad=\underset{t}{\lim } \operatorname{Hom}_{\mathscr{O}_{X}}\left(\pi_{*}\left(\mathscr{O}_{X^{\prime}} / I^{t t} \otimes \bigwedge^{q} \Omega_{X^{\prime} / X}\right) \otimes \mathscr{O}_{X} / I_{2}^{t}, \mathscr{T}\right) \\
\quad=\underset{t}{\lim } \operatorname{Hom}_{\mathscr{O}_{X}}\left(\pi_{*}\left(\mathscr{O}_{X^{\prime}} /\left(I^{\prime t}+\pi^{*} I_{2}^{t}\right) \otimes \bigwedge^{q} \Omega_{X^{\prime} / X}\right), \mathscr{T}\right) .
\end{aligned}
$$

But the chains of ideals $\left(I^{t t}+\pi^{*} I_{2}^{t}\right)_{t}$ and $\left(I_{2}^{t t}\right)_{t}$ are cofinal in each other.

We obtain exact sequences

$$
\begin{aligned}
0 & \rightarrow \underset{t}{\lim } \operatorname{Hom}_{\mathscr{O}_{X}}\left(\pi_{*}\left(\mathscr{O}_{X^{\prime}} / I_{2}^{\prime t} \otimes \bigwedge^{q} \Omega_{X^{\prime} / X}\right), \mathscr{T}\right) \\
& \rightarrow \underset{t}{\lim } \operatorname{Hom}_{\mathscr{O}_{X}}\left(\pi_{*}\left(\mathscr{O}_{X^{\prime}} / I^{\prime t} \otimes \bigwedge^{q} \Omega_{X^{\prime} / X}\right), \mathscr{T}\right) \\
& \rightarrow \underset{t}{\lim } \operatorname{Hom}_{\mathscr{C}_{X_{1}}}\left(\pi_{*}\left(\mathscr{O}_{X^{\prime}} / I^{\prime t} \otimes \bigwedge^{q} \Omega_{X^{\prime} / X}\right)\left|X_{1}, \mathscr{T}\right| X_{1}\right) \rightarrow 0 .
\end{aligned}
$$


In a similar but easier way, we obtain from Lemma 5.4 that

$$
\begin{aligned}
0 & \rightarrow \underset{t}{\lim } \operatorname{Hom}_{\mathscr{O}_{X}}\left(\mathscr{O}_{X} / I_{2}^{t}, \mathscr{T}\right) \\
& \rightarrow \underset{t}{\lim } \operatorname{Hom}_{\mathscr{O}_{X}}\left(\mathscr{O}_{X} / I^{t}, \mathscr{T}\right) \\
& \rightarrow \underset{t}{\lim } \operatorname{Hom}_{\mathscr{O}_{X_{1}}}\left(\mathscr{O}_{X} / I^{t}\left|X_{1}, \mathscr{T}\right| X_{1}\right) \rightarrow 0
\end{aligned}
$$

is exact.

We can combine these sequences into a diagram:

0

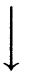

0

$\longleftarrow \underset{t}{\lim } \operatorname{Hom}_{\mathscr{O}_{X}}\left(\mathscr{O}_{X}\right.$

0

$\longleftarrow \stackrel{\lim }{\longrightarrow} \operatorname{Hom}_{\mathscr{O}_{X}}$

0

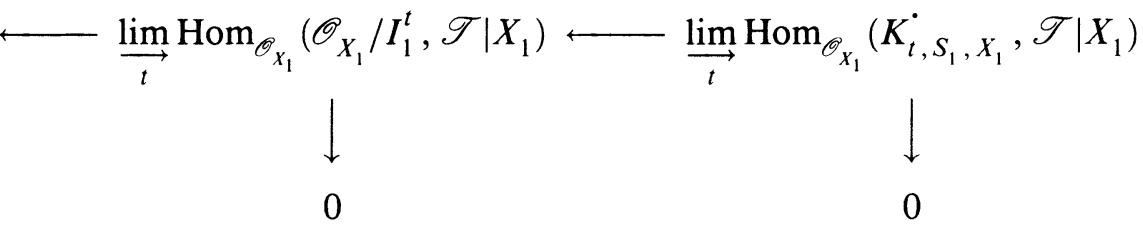

0 $\underset{t}{\lim } \operatorname{Hom}_{\mathscr{O}_{X}}\left(K_{t, S_{2}, X}^{\cdot}, \mathscr{T}\right)$ $\underset{t}{\lim } \operatorname{Hom}_{\mathscr{O}_{X}}\left(K_{t, S, X}^{\cdot}, \mathscr{T}\right)$

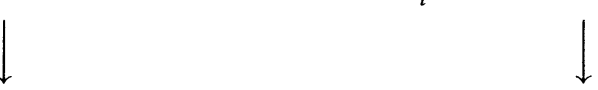

It follows now from our induction hypothesis that the middle complex is exact.

Proof of Theorem 5.1. We start with an injective resolution $0 \rightarrow \mathscr{M} \rightarrow \mathscr{T}$. We then obtain a double complex

$$
\underset{t}{\lim } \operatorname{Hom}\left(K_{t, S, X}^{\cdot}, \mathscr{T}^{\cdot}\right),
$$

which we think of as lying in the first quadrant such that the maps obtained from $K_{t, S, X}^{*}$ are horizontal, and such that the lower left-hand corner is

$$
\underset{t}{\lim } \operatorname{Hom}\left(K_{t, S, X}^{\operatorname{dim}\left(X^{\prime} / X\right)}, \mathscr{T}^{0}\right)
$$

To compute the homology of (12), we use the first filtration. By Lemma 5.2, we obtain the complex

$$
\Gamma_{S}\left(X, \mathscr{T}^{\cdot}\right)
$$

at horizontal position $\operatorname{dim}\left(X^{\prime} / X\right)$ and zeros everywhere else. The homology of (13) is clearly $H_{S}^{q}(X, \mathscr{M})$ at position $\left(\operatorname{dim}\left(X^{\prime} / X\right), q\right)$. Hence, (12) has homology $H_{S}^{q}(X, \mathscr{M})$ at position $q+\operatorname{dim}\left(X^{\prime} / X\right)$. 
To use the second filtration, we have to compute the homology of the complexes

$$
\operatorname{Hom}_{\mathscr{O}_{X}}\left(\pi_{*}\left(\mathscr{O}_{X^{\prime}} / I^{t-p^{\prime}} \otimes_{\mathscr{O}_{X^{\prime}}} \bigwedge^{p^{\prime}} \Omega_{X^{\prime} / X}\right), \mathscr{T}^{\cdot}\right),
$$

where $p^{\prime}=\operatorname{dim}\left(X^{\prime} / X\right)-p$.

We obtain

$$
\operatorname{Ext}_{\mathscr{O}_{X}}^{q}\left(\pi_{*}\left(\mathscr{O}_{X^{\prime}} / I^{\prime t-p^{\prime}} \otimes_{\mathscr{O}_{X^{\prime}}} \bigwedge^{p^{\prime}} \Omega_{X^{\prime} / X}\right), \mathscr{M}\right)
$$

which is by duality [6, Theorem III.11.1]

$$
\operatorname{Ext}_{\mathscr{O}_{X^{\prime}}}^{q+\operatorname{dim}\left(X^{\prime} / X\right)}\left(\mathscr{O}_{X^{\prime}} / I^{\prime t-p^{\prime}} \otimes_{\mathscr{O}_{X^{\prime}}} \bigwedge^{p^{\prime}} \Omega_{X^{\prime} / X}, \pi^{*} \mathscr{M} \otimes \omega_{X^{\prime} / X}\right)
$$

where we have used the fact that $R \pi_{*}=0$ on modules with support in $S^{\prime}$.

Simplifying (14) further, we obtain

$$
\begin{aligned}
& \operatorname{Ext}_{\mathscr{O}_{X^{\prime}}}^{q+\operatorname{dim}\left(X^{\prime} / X\right)}\left(\mathscr{O}_{X^{\prime}} / I^{\prime t-p^{\prime}} \otimes_{\mathscr{O}_{X^{\prime}}} \bigwedge^{p^{\prime}} \Omega_{X^{\prime} / X}, \pi^{*} \mathscr{M} \otimes \omega_{X^{\prime} / X}\right) \\
& =\operatorname{Ext}_{\mathscr{O}_{X^{\prime}}}^{q+\operatorname{dim}\left(X^{\prime} / X\right)}\left(\mathscr{O}_{X^{\prime}} / I^{\prime t-p^{\prime}}, \pi^{*} \mathscr{M} \otimes \omega_{X^{\prime} / X} \otimes\left(\bigwedge^{p^{\prime}} \Omega_{X^{\prime} / X}\right)^{*}\right) \\
& =\operatorname{Ext}_{\mathscr{O}_{X^{\prime}}}^{q+\operatorname{dim}\left(X^{\prime} / X\right)}\left(\mathscr{O}_{X^{\prime}} / I^{t t-p^{\prime}}, \pi^{*} \mathscr{M} \otimes \bigwedge^{\operatorname{dim}\left(X^{\prime} / X\right)-p^{\prime}} \Omega_{X^{\prime} / X}\right) .
\end{aligned}
$$

Hence, after taking homology for the second filtration in (12), we obtain a diagram with

$$
H_{S^{\prime}}^{q+\operatorname{dim}\left(X^{\prime} / X\right)}\left(X^{\prime}, \pi^{*} \mathscr{M} \otimes_{\mathscr{O}_{X^{\prime}}} \bigwedge^{p} \Omega_{X^{\prime} / X}\right)
$$

at position $(p, q)$. After reindexing, we obtain (7).

\section{ConstructibiLity}

In this section, we will use Theorem 5.1 to get some results on cohomology with support in the unstable locus. Roughly speaking, we will decompose the unstable locus as a union of a closed and a locally closed subvariety, which can be handled by Theorem 5.1. Then we use induction. It would be natural to try to use the well-known stratification into smooth subvarieties, due to Hesselink [7], Kirwan [9], and others. Unfortunately, this stratification turns out to be too fine for our purposes. The decomposition we must use is much coarser, and the parts are not necessarily smooth. What is worse, however, is that it does not always work! This leads us to a concept we call constructibility, which is introduced below.

As usual, $G$ will be a reductive algebraic group with a Borel subgroup $B \subset G$ containing a maximal torus $T \subset G$. $W$ will be a $G$-representation, and $R=$ $k[W]$. We define, furthermore, $X=\operatorname{Spec} R, X / G=\operatorname{Spec} R^{G}, d=\operatorname{dim} W$, $h=\operatorname{dim} R^{G}$. The roots of $B$ will be the negative roots, and $\Phi^{+}$will denote the set of positive roots. 
6.1. Reduction pairs and constructibility. In the sequel, a pair $(P, Y)$ will consist of a parabolic subgroup $P \subset G$ containing $B$ and a linear subspace $Y$ of $X$ which is preserved by $B$.

Definition 6.1.1. A reduction pair for $(P, Y)$ is a pair $\left(P_{1}, Y_{1}\right)$ such that

(1) $P_{1} \subset P, Y_{1} \subset Y$, and the inclusions are strict.

(2) $\left(P \backslash P_{1}\right) Y \cap Y \subset P_{1} Y_{1}$.

We first have to introduce some more notation: if $P$ is a parabolic subgroup of $G$, then $f: G \times{ }^{P} X \rightarrow G / P$ will be the natural projection map $\overline{(g, x)} \mapsto \bar{g}$.

If $P_{2} \supset P_{1}$ are parabolic subgroups of $G$, then for $l \geq 0$, we will denote with $\Omega_{\left[P_{2} / P_{1}\right]}^{l}$ the $\mathscr{O}_{G / P_{1}}$-module $\bigwedge^{l} \Omega_{\left(G / P_{1}\right) /\left(G / P_{2}\right)}$.

From the fact that there is a commutative diagram

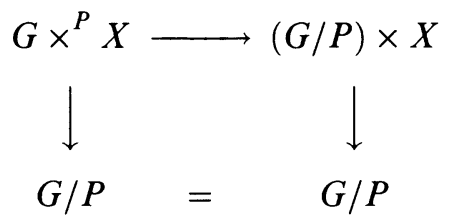

for any subgroup $P$ of $G$, we deduce that $\wedge^{l} \Omega_{\left(G \times{ }^{P_{1}} X\right) /\left(G \times{ }^{P_{2}} X\right)}=f^{*} \Omega_{\left[P_{2} / P_{1}\right]}^{l}$.

The quotient map $G / P_{1} \rightarrow G / P_{2}$ will be denoted by $\pi_{P_{2}}^{P_{1}}$. The same notation is used for the analogous map $G \times{ }^{P_{1}} X \rightarrow G \times{ }^{P_{2}} X$.

$l\left(P_{2} / P_{1}\right)$ will be the biggest $u$ such that there is a chain $P_{2}=P^{(u)} \supset P^{(u-1)} \supset$ $\cdots \supset P^{(0)}=P_{1}$ of parabolics, such that all inclusions are strict. Clearly, $l(G / B)$ is the rank of the semisimple part of $G$.

Finally, if $P_{u} \supset P_{u-1} \supset \cdots \supset P_{0}$ is a chain of parabolic subgroups of $G$, then we define $\Omega_{\left[P_{u} / P_{u-1} \cdots / P_{0}\right]}^{l_{u} \cdots l_{1}}$ for natural numbers $\left(l_{i}\right)_{i}$ as

$$
\Omega_{\left[P_{1} / P_{0}\right]}^{l_{1}} \otimes \pi_{P_{1}}^{P_{0}^{*}} \Omega_{\left[P_{2} / P_{1}\right]}^{l_{2}} \otimes \cdots \otimes \pi_{P_{u-1}}^{P_{0} *} \Omega_{\left[P_{u} / P_{u-1}\right]}^{l_{u}} .
$$

Lemma 6.1.2. Assume that $(P, Y)$ is a pair and that $\left(P_{1}, Y_{1}\right)$ is a reduction pair. Let $\mathscr{M}$ be a G-equivariant, quasicoherent $\mathscr{O}_{G \times{ }^{P} X}$-module. Then every $G$ representation that occurs in $H_{G \times{ }^{P} P Y}^{i}\left(G \times{ }^{P} X, \mathscr{M}\right)$ occurs in one of the following G-modules:

(1) $H_{G \times{ }^{P} P Y_{1}}^{i}\left(G \times{ }^{P} X, \mathscr{M}\right)$,

(2) $H_{G \times{ }^{P_{1} P_{1} Y}}^{i_{1}+2 \operatorname{dim}\left(P / P_{1}\right)}\left(G \times{ }^{P_{1}} X, \pi_{P}^{P_{1} *} \mathscr{M} \otimes f^{*} \Omega_{\left[P / P_{1}\right]}^{i_{2}}\right)$, where $i_{1}+i_{2}=i$,

(3) $H_{G \times{ }^{P_{1}} P_{1} Y_{1}}^{i_{1}+1+2 \operatorname{dim}\left(P / P_{1}\right)}\left(G \times{ }^{P_{1}} X, \pi_{P}^{P_{1} *} \mathscr{M} \otimes f^{*} \Omega_{\left[P / P_{1}\right]}^{i_{2}}\right)$, where $i_{1}+i_{2}=i$.

Proof. By the standard long exact sequence for cohomology with support, any representation occurring in $H_{G \times{ }^{P} P Y}^{i}\left(G \times{ }^{P} X, \mathscr{M}\right)$ occurs in $H_{G \times{ }^{P} P Y_{1}}^{i}\left(G \times{ }^{P} X, \mathscr{M}\right)$ also or in

$$
H_{G \times{ }^{P}\left(P Y \backslash P Y_{1}\right)}^{i}\left(G \times{ }^{P}\left(X \backslash P Y_{1}\right), \mathscr{M}\right)
$$


By (1), we have to concern ourselves with only the latter case. We first prove a sublemma.

Lemma 6.1.3. The commutative diagram

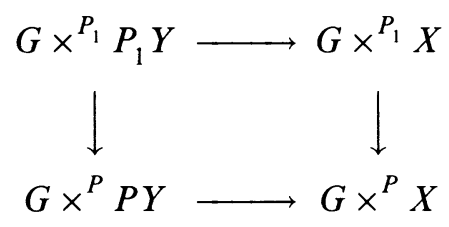

restricts to a diagram

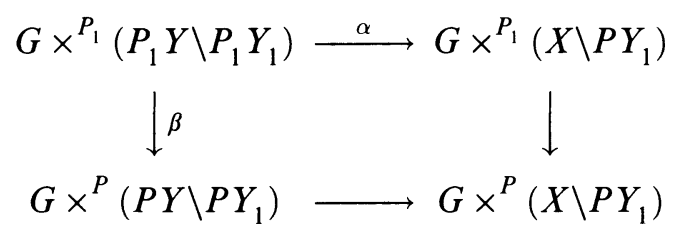

where $\alpha$ is a closed immersion and $\beta$ is a bijection.

Proof. This can be deduced from (2) in Definition 6.1.1. We first show that $Y \backslash P Y_{1}=Y \backslash P_{1} Y_{1}$ which is equivalent to $Y \cap P Y_{1}=Y \cap P_{1} Y_{1}$.

$$
\begin{aligned}
Y \cap P Y_{1} & =\left(Y \cap P_{1} Y_{1}\right) \cup\left(Y \cap\left(P \backslash P_{1}\right) Y\right) \\
& =Y \cap P_{1} Y_{1} .
\end{aligned}
$$

From this, it follows that $\alpha$ and $\beta$ are defined. For $\alpha$, we have to show that $P_{1} Y \backslash P_{1} Y_{1}$ is a closed subset of $X \backslash P Y_{1}$. But

$$
\begin{aligned}
P_{1} Y \backslash P_{1} Y_{1} & =P_{1}\left(Y \backslash P_{1} Y_{1}\right)=P_{1}\left(Y \backslash P Y_{1}\right) \\
& =P_{1} Y \backslash P Y_{1} \subset X \backslash P Y_{1},
\end{aligned}
$$

and the last inclusion is closed.

Similarly, for $\beta$, we first have to show that $P\left(P_{1} Y \backslash P_{1} Y_{1}\right) \subset P Y \backslash P Y_{1}$. Again,

$$
P\left(P_{1} Y \backslash P_{1} Y_{1}\right)=P\left(Y \backslash P_{1} Y_{1}\right)=P\left(Y \backslash P Y_{1}\right)=P Y \backslash P Y_{1} \text {. }
$$

This also shows that $\beta$ is surjective.

To show that $\beta$ is a bijection, let $y \in Y \backslash P Y_{1}$. Any other element in $G \times{ }^{P}$ $\left(P Y \backslash P Y_{1}\right)$ is in the $G$-orbit of such an element. A quick check then shows $(\cong$ means "there is a bijection")

$$
\beta^{-1}(y) \cong\left\{\left(p, y^{\prime}\right) \in P \times\left(P_{1} Y \backslash P_{1} Y_{1}\right) \mid p y^{\prime}=y\right\} / P_{1} .
$$

Hence

$$
\begin{aligned}
\beta^{-1}(y) & \cong\left\{p \in P \mid p^{-1} y \in P_{1} Y \backslash P_{1} Y_{1}\right\} / P_{1} \\
& \cong\left\{p \in P \mid p^{-1} y \in P_{1} Y \backslash P Y_{1}\right\} / P_{1} \quad \text { (as above) } \\
& \cong\left\{p \in P \mid y \in p P_{1} Y \backslash P Y_{1}\right\} / P_{1} \\
& \cong\left\{p \in P \mid y \in p P_{1} Y\right\} / P_{1} \quad \text { (since } y \notin P Y_{1} \text { by hypothesis.) } \\
& \cong \text { singleton (using (2) in Definition 6.1.1). }
\end{aligned}
$$


Remark 6.1.4. We can actually prove that the existence of diagram (16), together with the fact that $\beta$ is a bijection, is equivalent to condition (2) in Definition 6.1.1.

Now we continue with the proof of Lemma 6.1.2.

By Theorem 5.1 and diagram (16), every representation that occurs in (15) must occur in one of the representations

$$
H_{G \times P_{1}\left(P_{1} Y \backslash P_{1} Y_{1}\right)}^{i_{1}+2 \operatorname{dim}\left(P / P_{1}\right)}\left(G \times P^{P_{1}}\left(X \backslash P Y_{1}\right), \pi_{P}^{P_{1}^{*}} \mathscr{M} \otimes f^{*} \Omega_{\left[P / P_{1}\right]}^{i_{2}}\right),
$$

where $i_{1}+i_{2}=i$.

But $G \times{ }^{P_{1}}\left(X \backslash P_{1} Y_{1}\right)$ is an open subset of $G \times{ }^{P_{1}} X$ containing $G \times{ }^{P_{1}}\left(X \backslash P Y_{1}\right)$, and $G \times{ }^{P_{1}}\left(P_{1} Y \backslash P_{1} Y_{1}\right)$ is still a closed subset of $G \times{ }^{P_{1}}\left(X \backslash P_{1} Y_{1}\right)$. Therefore, (17) is equal to (excision)

$$
H_{G \times P_{1}\left(P_{1} Y \backslash P_{1} Y_{1}\right)}^{i_{1}+2 \operatorname{dim}\left(P / P_{1}\right)}\left(G \times P^{P_{1}}\left(X \backslash P_{1} Y_{1}\right), \pi_{P}^{P_{1}^{*}} \mathscr{M} \otimes f^{*} \Omega_{\left[P / P_{1}\right]}^{i_{2}}\right)
$$

Invoking again the long exact cohomology sequence yields that any representation occurring in (18) must occur in

$$
H_{G \times{ }^{P_{1}} P_{1} Y}^{i_{1}+2 \operatorname{dim}\left(P / P_{1}\right)}\left(G \times{ }^{P_{1}} X, \pi_{P}^{P_{1} *} \mathscr{M} \otimes f^{*} \Omega_{\left[P / P_{1}\right]}^{i_{2}}\right)
$$

or in

$$
H_{G \times{ }^{P_{1}} P_{1} Y_{1}}^{i_{1}+1+2 \operatorname{dim}\left(P / P_{1}\right)}\left(G \times{ }^{P_{1}} X, \pi_{P}^{P_{1} *} \mathscr{M} \otimes f^{*} \Omega_{\left[P / P_{1}\right]}^{i_{2}}\right)
$$

Definition 6.1.5. A pair $(P, Y)$ is constructible if and only if one of the following holds.

(1) $P Y=Y$ and $Y=X_{\lambda}$ for some $\lambda \in Y(T)$ (including 0 ) belonging to the Weyl chamber determined by $B$.

(2) There exists a reduction pair $\left(P_{1}, Y_{1}\right)$ for $(P, Y)$ such that $\left(P_{1}, Y_{1}\right)$, $\left(P_{1}, Y\right)$, and $\left(P, Y_{1}\right)$ are constructible.

Proposition 6.1.6. Assume that the pair $(G, Y)$ is constructible. Then any $G$ representation occuring in $H_{G Y}^{i}\left(X, \mathscr{O}_{X}\right)$ occurs in some

$$
H_{G \times{ }^{B} Y^{\prime}}^{j}\left(G \times{ }^{B} X, f^{*} \pi_{P^{\prime}}^{B *} \Omega_{\left[P_{u} / P_{u-1} / \cdots / P_{0}\right]}^{i_{u} \cdots i_{1}}\right),
$$

where

(1) $\left(P^{\prime}, Y^{\prime}\right)$ is a pair such that $P^{\prime} \subset G, Y^{\prime} \subset Y, P Y^{\prime}=Y^{\prime}, Y^{\prime}=X_{\lambda}$, and $\lambda$ is in the Weyl chamber determined by $B$.

(2) $P_{u} \supset P_{u-1} \supset \cdots \supset P_{0}$ is a chain (with strict inclusions) such that $G=P_{u}$, $P^{\prime}=P_{0}$.

(3) $i \leq j+i_{1}+\cdots+i_{u} \leq i+u+2 \operatorname{dim}\left(G / P^{\prime}\right)$.

Proof. This follows by induction from Lemma 6.1.2 and the observation that $H_{G \times P^{P^{\prime}} Y^{\prime}}^{\cdot}\left(G \times P^{P^{\prime}} X, f^{*} \Omega_{\left[P_{u} / P_{u-1} / \cdots / P_{0}\right]}^{i_{u} \cdots i_{1}}\right)=H_{G \times{ }^{B} Y^{\prime}}^{\cdot}\left(G \times{ }^{B} X, f^{*} \pi_{P^{\prime}}^{B *} \Omega_{\left[P_{u} / P_{u-1} / \cdots / P_{0}\right]}^{i_{u} \cdots i_{1}}\right)$.

Proposition 6.1.7. Let $(G, Y)$ be a constructible pair, and let $\chi \in X(T)$ be a dominant character (with respect to $\left.\Phi^{+}\right)$. Assume that $\chi-w\left(\sum_{\rho \in S} \rho\right.$ ) is 
strongly critical for $(T, W)$, for all $w \in \mathscr{W}_{G}, S \subset-\Phi^{+}$. Then there will be no G-representation with highest weight $\chi$ in $H_{G Y}^{i}\left(X, \mathscr{O}_{X}\right)$ for $0 \leq i \leq$ $d-l(G / B)-2 \operatorname{dim}(G / B)-1$.

Proof. By Proposition 6.1.6, it is sufficient to prove the same statement for

$$
H_{G \times{ }^{B} Y^{\prime}}^{j}\left(G \times{ }^{B} X, f^{*} \pi_{P^{\prime}}^{B *} \Omega_{\left[P_{u} / P_{u-1} / \cdots / P_{0}\right]}^{i_{u} \cdots i_{1}}\right),
$$

where

$$
\begin{aligned}
0 & \leq j+i_{u}+\cdots+i_{1} \\
& \leq d-l(G / B)-2 \operatorname{dim}(G / B)-1+u+2 \operatorname{dim}\left(G / P^{\prime}\right) \\
& \leq d-1 .
\end{aligned}
$$

This is obviously true if $Y^{\prime}=\{0\}$. Hence, we assume that $Y^{\prime} \neq\{0\}$.

(19) is equal to

$$
H_{G \times{ }^{B} Y^{\prime}}^{j}\left(G \times{ }^{B} X, \mathscr{O}_{G \times{ }^{B} X} \otimes_{\mathscr{O}_{G / B}} \pi_{P^{\prime}}^{B *} \Omega_{\left[P_{u} / \cdots / P_{0}\right]}^{i_{u} \cdots i_{1}}\right) .
$$

If we take the fiber of $\pi_{P^{\prime}}^{B *} \Omega_{\left[P_{u} / \cdots / P_{0}\right]}^{i_{u} \cdots i_{1}}$ over $[B]$, then we obtain

$$
\bigwedge^{i_{u}}\left(\mathbf{p}_{u} / \mathbf{p}_{u-1}\right)^{*} \otimes \cdots \otimes \bigwedge^{i_{1}}\left(\mathbf{p}_{1} / \mathbf{p}_{0}\right)^{*}
$$

where $\mathbf{p}_{j}$ is the Lie algebra of $P_{j}$.

(20) is a $B$-representation which has a filtration whose associated quotient representations are $T$-characters of the form

$$
\chi_{1}^{(S)}=\sum_{\rho \in S} \rho, \quad \text { where } S \subset-\Phi^{+} .
$$

Using Lemma 6.2 .1 of the next section, it is sufficient that $\chi-w \chi_{1}^{(S)}$ is strongly critical for $(T, W)$ for all $w \in \mathscr{W}_{G}$ and for all $S \subset-\Phi^{+}$. But this was exactly the hypothesis.

It is well known that every fiber of $X \rightarrow X / G$ contains a unique closed orbit. A point $x \in X$ is called stable if for all $\lambda \in Y(G)$ neither $\lim _{t \rightarrow 0} \lambda(t) x$ nor $\lim _{t \rightarrow \infty} \lambda(t) x$ exists. Stable points have finite stabiliser and closed $G$-orbit. They form an invariant open subset of $X$.

Hence, we deduce that $\operatorname{dim} X=\operatorname{dim}(X / G)+\operatorname{dim} G$ if there is at least one stable point in $X$.

Let us also recall the following theorem.

Theorem 6.1.8 [15]. Assume that a semisimple group $G$ acts on an affine variety $X$ with factorial coordinate ring such that the generic stabiliser is finite. Then $X$ has a stable point.

To simplify the notation a bit, we will say that $X^{u}$ is constructible if it is of the form $G X_{\lambda}$, where $\left(G, X_{\lambda}\right)$ is a constructible pair. 
Theorem 6.1.9. Let $G$ be semisimple, and assume that $X^{u}$ is constructible. Assume, furthermore, that $X$ has a G-stable point. Then Conjecture $3.4^{\prime}$ is true.

Proof. Let $\chi$ be a character of $G$, and let $\chi_{1} \in X(T)$ be the highest weight of its corresponding $G$-representation. The hypotheses for Conjecture $3.4^{\prime}$ imply that $\chi_{1}-w\left(\sum_{\rho \in S} \rho\right)$ will be strongly critical for $(T, W)$ for all $w \in \mathscr{W}_{G}$, $S \subset-\Phi^{+}$. Hence, by Proposition 6.1.7, there will be no representation with character $\chi$ in $H_{X^{u}}^{i}\left(X, \mathscr{O}_{X}\right)$ for $0 \leq i \leq d-l(G / B)-2 \operatorname{dim}(G / B)-1$.

However, since $G$ is assumed to be semisimple and $X$ has a stable point, $l(G / B)+2 \operatorname{dim}(G / B)=\operatorname{dim} G$, and $d-\operatorname{dim} G-1=h-1$. Hence, the conditions for Corollary 4.2 are satisfied, and, therefore, $R_{\chi}^{G}$ is Cohen-Macaulay.

Remark 6.1.10. Note that in Proposition 6.1.7, we actually prove more than in Theorem 6.1.9. However, the author has no example of where this makes any difference.

Theorem 6.1.11. Conjecture $3.4^{\prime}$ is true in the case $G=S L(V), \operatorname{dim} V=2$.

Proof. We may assume that $W$ does not contain trivial representations.

Assume first that $W=V$ or $W=S^{2} V$. Then $k[W]^{G}$ is a PID. Hence, $R_{\chi}^{G}$ is a torsion free module over a PID, and, therefore, Cohen-Macaulay. This means that Conjecture $3.4^{\prime}$ is vacuous and, hence, true.

Assume now that $W \neq V$ and $W \neq S^{2} V$. Then $X$ has a stable point by Theorem 6.1.8. From the fact that $X(T)=\mathbf{Z}$, we deduce that $X^{u}=G X_{\lambda}$, where

$$
\lambda(z)=\left(\begin{array}{cc}
z & 0 \\
0 & z^{-1}
\end{array}\right) .
$$

From Definition 6.1.1 or else from (26) below, we deduce that $(B,\{0\})$ is a reduction pair for $\left(G, X_{\lambda}\right)$. Since $\left(B, X_{\lambda}\right),(G,\{0\})$, and $(B,\{0\})$ are constructible by the first condition of Definition 6.1.5, we deduce that $\left(G, X_{\lambda}\right)$ is constructible.

6.2. Some computations. The following lemma was used in the proof of Proposition 6.1.7. This subsection will be devoted to its proof.

Lemma 6.2.1. Assume that $\lambda \in Y(T)$ belongs to the Weyl chamber determined by $B$. Let $\chi, \chi_{1} \in X(T)$, where $\chi$ is dominant and $\chi-w \chi_{1}$ is strongly critical for $(T, W)$, for all $w \in \mathscr{W}_{G}$. Assume, furthermore, that $X$ has a stable point. Then no G-representation with highest weight $\chi$ occurs in

$$
H_{G \times{ }^{B} X_{\lambda}}^{\cdot}\left(G \times{ }^{B} X, \mathscr{O}_{G \times{ }^{B} X} \otimes_{\mathscr{O}_{G / B}} \tilde{\chi}_{1}\right) .
$$

Proof. Let $Y=X_{\lambda}$. There is a spectral sequence

$$
\begin{aligned}
E_{2}^{p q}: & H^{p}\left(\mathscr{H}_{G \times{ }^{B} Y}^{q}\left(G \times{ }^{B} X, \mathscr{O}_{G \times{ }^{B} X} \otimes_{\mathscr{O}_{G / B}} \tilde{\chi}_{1}\right)\right) \\
& \Rightarrow H_{G \times{ }^{B} Y}^{p+q}\left(G \times{ }^{B} X, \mathscr{O}_{G \times{ }^{B} X} \otimes_{\mathscr{O}_{G / B}} \tilde{\chi}_{1}\right) .
\end{aligned}
$$


Furthermore, it is easy to see that

$$
\begin{aligned}
\mathscr{H}_{G \times{ }^{B} Y}^{q}\left(G \times{ }^{B} X, \mathscr{O}_{G \times{ }^{B} X} \otimes_{\mathscr{O}_{G / B}} \tilde{\chi}_{1}\right) & =\mathscr{H}_{G \times{ }^{B} Y}^{q}\left(G \times{ }^{B} X,\left(\mathscr{O}_{X} \otimes_{k} \chi_{1}\right)^{\sim}\right) \\
& =\left(H_{Y}^{q}\left(X, \mathscr{O}_{X}\right) \otimes_{k} \chi_{1}\right)^{\sim}
\end{aligned}
$$

as $\mathscr{O}_{G / B}$-modules.

Let $J$ be the defining ideal of $Y . J$ is generated by a subspace $W^{\prime}$ of $W$. Define $W^{\prime \prime}=W / W^{\prime}, d^{\prime}=\operatorname{dim} W^{\prime}$. We need the following result.

Lemma 6.2.2. $H_{J}^{i}(R)=0$ if $i \neq d^{\prime}$.

(2) $H_{J}^{d^{\prime}}(R)$ is, as a T-representation, isomorphic to

$$
\left(\bigwedge^{d^{\prime}} W^{\prime}\right)^{*} \otimes \bigoplus_{n=0}^{\infty} S^{n}\left(W^{*} \oplus W^{\prime \prime}\right) .
$$

Proof. The first statement is clear since $J$ is generated by a system of parameters.

For the second statement, we use the fact that $H_{J}^{d^{\prime}}(R)=\underline{\lim }_{t} \operatorname{Ext}_{R}\left(R / J^{t}, R\right)$. We first compute

$$
\operatorname{Ext}_{R}\left(J^{t} / J^{t+1}, R\right) \cong\left(S^{t} W^{\prime}\right)^{*} \otimes \operatorname{Ext}_{R}^{i}(R / J, R) .
$$

Again $\operatorname{Ext}^{i}(R / J, R)=0$ if $i \neq d^{\prime}$. On the other hand, using the Koszul resolution for $R / J$, we easily compute that

$$
\operatorname{Ext}_{R}^{d^{\prime}}(R / J, R) \cong\left(\bigwedge^{d^{\prime}} W^{\prime}\right)^{*} \otimes R / J
$$

Hence, as $T$-module,

$$
\begin{aligned}
H_{J}^{i}(R) & =\bigoplus_{t} \bigoplus_{t^{\prime}}\left(\bigwedge^{d^{\prime}} W^{\prime}\right)^{*} \otimes\left(S^{t} W^{\prime}\right)^{*} \otimes S^{t^{\prime}} W^{\prime \prime} \\
& =\left(\bigwedge^{d^{\prime}} W^{\prime}\right)^{*} \otimes \bigoplus_{t \geq 0} S^{t}\left(W^{\prime *} \oplus W^{\prime \prime}\right) . \quad
\end{aligned}
$$

Now assume that $W=\alpha_{1} \oplus \alpha_{2} \oplus \cdots \oplus \alpha_{d}$ as $T$-representation, where $\alpha_{i} \in$ $X(T)$. Define $I=\left\{i \in\{1, \ldots, d\} \mid\left\langle\lambda, \alpha_{i}\right\rangle \geq 0\right\}$. Then by construction, the weights of $W^{\prime}$ and $W^{\prime \prime}$ are resp. $\left(\alpha_{i}\right)_{i \in I}$ and $\left(\alpha_{i}\right)_{i \notin I}$. Thus, $H_{Y}^{d^{\prime}}\left(X, \mathscr{O}_{X}\right) \otimes \chi_{1}$ has a filtration (as $B$-representation) whose associated graded quotients $\chi^{\prime}$ are by Lemma 6.2.2 of the form

$$
-\sum_{i \in I} \alpha_{i}-\sum_{i \in I} a_{i} \alpha_{i}+\sum_{i \notin I} b_{i} \alpha_{i}+\chi_{1}
$$

where $a_{i}, b_{i} \in \mathbf{N}$.

Hence, they have the property that

$$
\begin{aligned}
\left\langle\lambda, \chi^{\prime}-\chi_{1}\right\rangle & =\left\langle\lambda,-\sum_{i \in I} \alpha_{i}\right\rangle-\sum_{i \in I} a_{i}\left\langle\lambda, \alpha_{i}\right\rangle+\sum_{i \notin I} b_{i}\left\langle\lambda, \alpha_{i}\right\rangle \\
& \leq-\sum_{i \in I}\left\langle\lambda, \alpha_{i}\right\rangle .
\end{aligned}
$$

Let $\bar{\rho} \in X(T)_{\mathbf{Q}}$ be half the sum of the positive roots. 
Now assume that $\chi$ does occur in (21). Then it must also occur somewhere in the $E_{2}$-term of the spectral sequence (22). Hence, by Bott's Theorem [3] and by (23), it must be of the form $w\left(\chi^{\prime}+\bar{\rho}\right)-\bar{\rho}$, where $w \in \mathscr{W}_{G}$, and $\chi^{\prime}$ is of the form (24).

From (25), we deduce

$$
\begin{aligned}
-\sum_{i \in I}\left\langle\lambda, \alpha_{i}\right\rangle & \geq\left\langle\lambda, w^{-1}(\chi+\bar{\rho})-\bar{\rho}-\chi_{1}\right\rangle \\
& =\left\langle\lambda, w^{-1}\left(\chi-w \chi_{1}\right)+w^{-1} \bar{\rho}-\bar{\rho}\right\rangle \\
& =\left\langle\lambda, w^{-1}\left(\chi-w \chi_{1}\right)\right\rangle+\left\langle\lambda, w^{-1} \bar{\rho}-\bar{\rho}\right\rangle .
\end{aligned}
$$

However, by Lemma 6.2 .3 below and by the hypothesis, $w^{-1}\left(\chi-w \chi_{1}\right)$ is strongly critical for $(T, W)$. Hence, we will deduce that (Lemma 6.2.4) $\left\langle\lambda, w^{-1}\left(\chi-w \chi_{1}\right)\right\rangle>-\sum_{i \in I}\left\langle\lambda, \alpha_{i}\right\rangle$.

Since, furthermore, $\left\langle\lambda, w^{-1} \bar{\rho}-\bar{\rho}\right\rangle \geq 0$ (Lemma 6.2.5), we obtain a contradiction.

Now we will fill in the few missing steps in Lemma 6.2.2.

Lemma 6.2.3. If $\chi$ is strongly critical for $(T, W)$ and $w \in \mathscr{W}_{G}$, then $w(\chi)$ is also strongly critical for $(T, W)$.

Proof. True, because $\mathscr{W}_{G}$ permutes the weights of $W$.

Lemma 6.2.4. Assume that $\chi$ is strongly critical for $(T, W)$ and $\lambda \in Y(T)$. Assume, furthermore, that $X$ has a stable point. Define

$$
I=\left\{i \in\{1, \ldots, d\} \mid\left\langle\lambda, \alpha_{i}\right\rangle \geq 0\right\} .
$$

Then $\langle\lambda, \chi\rangle>-\sum_{i \in I}\left\langle\lambda, \alpha_{i}\right\rangle$.

Proof. The fact that $X$ has a stable point implies that there exists an $i$ such that $\left\langle\lambda, \alpha_{i}\right\rangle>0$. By definition, $\chi=\sum_{i=1}^{d} a_{i} \alpha_{i},-1<a_{i} \leq 0$. Hence,

$$
\begin{aligned}
\langle\lambda, \chi\rangle & =\sum_{i \in I} a_{i}\left\langle\lambda, \alpha_{i}\right\rangle+\sum_{i \notin I} a_{i}\left\langle\lambda, \alpha_{i}\right\rangle \\
& >-\sum_{i \in I}\left\langle\lambda, \alpha_{i}\right\rangle \cdot
\end{aligned}
$$

Lemma 6.2.5. Let $\lambda \in Y(T)$ belong to the Weyl chamber determined by $B$. Let $\bar{\rho}$ be as in the proof of Lemma 6.2.1. Then $\langle\lambda, w \bar{\rho}-\bar{\rho}\rangle \geq 0$ for all $w \in \mathscr{W}_{G}$.

Proof. Since $\lambda$ belongs to the Weyl chamber determined by $B$, we deduce easily that $\langle\lambda, \rho\rangle \leq 0$ for all $\rho \in \Phi^{+}$. On the other hand, the definition of $\bar{\rho}$ immediately implies that $w \bar{\rho}-\bar{\rho}$ is a sum of negative roots.

6.3. A combinatorial criterion for constructibility. To verify whether a pair $\left(P_{1}, Y_{1}\right)$ is a reduction pair for some other pair $(P, Y)$, we need some way of checking condition (2) in Definition 6.1.1. A simple criterion that can be checked on the weights of $W$ is given below. 
Proposition 6.3.1. Assume that $\left(P_{1}, Y_{1}\right),(P, Y)$ are pairs such that $P_{1} \subset P$, $Y_{1} \subset Y$, and the inclusions are strict. If

$$
\forall w \in \mathscr{W}_{P} \backslash \mathscr{W}_{P_{1}}: w Y \cap Y \subset \bigcup_{w^{\prime} \in \mathscr{W}_{P_{1}}} w^{\prime} Y_{1}
$$

then $\left(P_{1}, Y_{1}\right)$ is a reduction pair for $(P, Y)$.

Proof. Assume that (26) holds. By the Bruhat decomposition,

$$
P_{1}=B \mathscr{W}_{P_{1}} B
$$

and

Hence,

$$
P \backslash P_{1}=B\left(\mathscr{W}_{P} \backslash \mathscr{W}_{P_{1}}\right) B
$$

$$
\begin{aligned}
\left(P \backslash P_{1}\right) Y \cap Y & =B\left(\mathscr{W}_{P} \backslash \mathscr{W}_{P_{1}}\right) Y \cap Y \\
& =B\left(\left(\mathscr{W}_{P} \backslash \mathscr{W}_{P_{1}}\right) Y \cap Y\right) \\
& \subset B \mathscr{W}_{P_{1}} Y_{1} \\
& =P_{1} Y_{1} \cdot \square
\end{aligned}
$$

\section{THE CASE OF MATRIX CONCOMITTANTS}

In this section, we will verify the major hypothesis of Theorem 6.1 .8 for $\mathbf{T}_{m, n}$, namely, that $X^{u}$ is constructible. Using (26), this can be done combinatorially. As a consequence, we obtain that $\mathbf{T}_{m, n}$ is Cohen-Macaulay in general (Theorem 7.3.6).

We define $G=S L(V)$, where $\operatorname{dim} V=n, W=\operatorname{End}(V)^{m *}$, and $X=$ Spec $k[W] . T \subset G$ will be a maximal torus. We will choose a basis in $V$ such that the action of $T$ on $V$ is diagonal, i.e., of the form $\operatorname{diag}\left(z_{1}, \ldots, z_{n}\right)$, where $z_{i} \in k$ and $z_{1} \cdots z_{n}=1$.

7.1. Ordered partitions. If $n$ is an integer, then an ordered partition $\nu$ of $n$ will be a tuple $\left(\nu_{1}, \nu_{2}, \ldots, \nu_{u}\right)$ such that $\sum_{i=1}^{u} \nu_{i}=n, \nu_{i} \in \mathbf{N}_{0}$. If $\nu$ is an ordered partition of some unspecified number, then that number will be denoted by $\sum \nu_{i}$. We will also use the empty tuple ( ) as the unique ordered partition of 0 .

If $\left(\nu^{(i)}\right)_{i=1, \ldots, v}$ are ordered partitions, where $\nu^{(i)}=\left(\nu_{1}^{(i)}, \ldots, \nu_{u_{i}}^{(i)}\right)$, then $\left(\nu^{(1)}, \ldots, \nu^{(v)}\right)$ is the ordered partition $\left(\nu_{1}^{(1)}, \ldots, \nu_{u_{1}}^{(1)}, \nu_{1}^{(2)}, \ldots, \nu_{u_{v}}^{(v)}\right)$.

If $\eta, \nu$ are two ordered partitions, then we say that $\eta$ is a refinement of $\nu$ (notation: $\eta \triangleleft \nu)$ if $\eta=\left(\eta^{(1)}, \ldots, \eta^{(v)}\right)$, where the $\eta^{(i)}$ are ordered partitions and $\nu=\left(\sum \eta^{(1)}, \ldots, \sum \eta^{(v)}\right)$.

The ordered partitions of $n$, ordered by $\triangleleft$, form a partially ordered set with minimal element $\eta_{\min }=(1, \ldots, 1)$ and maximal element $\eta_{\max }=(n)$.

Let $B$ be the Borel subgroup of $G$ consisting of the upper triangular matrices. Then any $\lambda \in Y(T)$ belonging to the Weyl chamber determined by $B$ will be of the form

$$
z \rightarrow \operatorname{diag}(\underbrace{z^{i_{1}}, \ldots, z^{i_{1}}}_{\eta_{1} \text { times }}, \underbrace{z^{i_{2}}, \ldots, z^{i_{2}}}_{\eta_{2} \text { times }}, \ldots, \underbrace{z^{i_{u}}, \ldots, z^{i_{u}}}_{\eta_{u} \text { times }}),
$$


where $i_{1}>i_{2}>\cdots>i_{u}$ and $\eta_{1} i_{1}+\eta_{2} i_{2}+\cdots+\eta_{u} i_{u}=0$. With a slight abuse of notation, we will denote this one-parameter subgroup by $\lambda_{\eta}$, where $\eta$ is the ordered partition of $n$ given by $\left(\eta_{1}, \ldots, \eta_{u}\right)$. This notation is justified in this context by the fact that if $\lambda_{1}, \lambda_{2} \in Y(T)$ are of the form (27) with the same numbers $\eta_{1}, \ldots, \eta_{u}$, then $G\left(\lambda_{1}\right)=G\left(\lambda_{2}\right)$ and $X_{\lambda_{1}}=X_{\lambda_{2}}$.

Clearly, $B=G\left(\lambda_{\eta_{\min }}\right)$ and $G=G\left(\lambda_{\eta_{\max }}\right)$.

Lemma 7.1.1. If $\eta \triangleleft \nu$, then

(1) $G\left(\lambda_{\eta}\right) \subset G\left(\lambda_{\nu}\right)$.

(2) $X_{\lambda_{\eta}} \supset X_{\lambda_{\nu}}$.

(3) $G\left(\lambda_{\eta}\right) X_{\lambda_{\nu}}=X_{\lambda_{\nu}}$

Proof. This follows by inspection.

Lemma 7.1.2. $X^{u}=G X_{\lambda_{\eta_{\min }}}$.

Proof. This follows by Lemmas 7.1.1 and 4.3.

Now define $Q=\{1, \ldots, n\}$. If $\nu$ is an ordered partition of $n$, then $Q_{\nu}$ will be the partition of $Q$ given by $\left\{\left\{1, \ldots, \nu_{1}\right\},\left\{\nu_{1}+1, \ldots, \nu_{1}+\nu_{2}\right\}, \ldots,\left\{\nu_{1}+\right.\right.$ $\left.\left.\cdots+\nu_{u-1}, \ldots, n\right\}\right\}$. The elements of $Q_{\nu}$ will be indexed as $Q_{\nu, i}$, where $Q_{\nu, i}=\left\{\nu_{1}+\cdots+\nu_{i-1}, \ldots, \nu_{1}+\cdots+\nu_{i}\right\}$.

If $T$ is an arbitrary set, then $S_{T}$ will be the permutation group of $T$. If $Q_{\nu}$ is a partition of $Q$, then $S_{Q_{\nu}}=\prod_{T \in Q_{\nu}} S_{T} \cdot Q_{\nu}^{\circ}$ will be the set $\bigcup_{j>i} Q_{\nu, i} \times$ $Q_{\nu, j} \subset Q \times Q$.

It is easily verified that if $\nu$ is an ordered partition, then

$$
\mathscr{W}_{P\left(\lambda_{\nu}\right)}=S_{Q_{\nu}},
$$

and the weights of $X_{\lambda_{\nu}}$ (considered as a subspace of $W^{*}$ ) are

$$
\left\{\left(z_{i} z_{j}^{-1}\right)_{(i, j) \in Q_{\nu}^{\circ}}\right. \text {. }
$$

Lemma 7.1.3. Let $\left(\eta_{1}, \nu_{1}\right),(\eta, \nu)$ be pairs of ordered partitions. Assume that $\eta_{1} \triangleleft \eta, \nu \triangleleft \nu_{1}$, and $\eta_{1} \neq \eta, \nu_{1} \neq \nu$. Suppose that

$$
\forall w \in S_{Q_{\eta}} \backslash S_{Q_{\eta_{1}}}: \exists w^{\prime} \in S_{Q_{\eta_{1}}}: w Q_{\nu}^{\circ} \cap Q_{\nu}^{\circ} \subset w^{\prime} Q_{\nu_{1}}^{\circ} .
$$

Then $\left(G\left(\lambda_{\eta_{1}}\right), X_{\lambda_{\nu_{1}}}\right)$ is a reduction pair for $\left(G\left(\lambda_{n}\right), X_{\lambda_{\nu}}\right)$.

Proof. This is just a translation of (26) to the present situation using (28) and (29).

7.2. Set-theoretic computations. In this subsection, we will verify (30) for certain special pairs of partitions.

For the sequel, let $\eta=\left(\eta^{(1)}, \eta^{(2)}, x\right), \nu=\left(\nu^{(1)}, a, 1, \nu^{(3)}\right)$ be fixed ordered partitions of $n$ where

(a) $\eta^{(1)}, \eta^{(2)}, \nu^{(1)}, \nu^{(3)}$ are ordered partitions.

(b) $x, a \in \mathbf{N}_{0}$.

(c) $\eta^{(1)} \triangleleft \nu^{(1)}$. 
(d) $\nu^{(3)}$ consists entirely of ones.

(e) $x+a>n-\sum \nu^{(1)}$.

Then we define $\eta_{1}=\left(\eta^{(1)}, \eta^{(2)}, x-n+\sum \nu^{(1)}+a, n-\sum \nu^{(1)}-a\right), \nu_{1}=$ $\left(\nu^{(1)}, a+1, \nu^{(3)}\right)$. To simplify the notation, we write $K=Q_{\eta}, K_{1}=Q_{\eta_{1}}$, $L=Q_{\nu}, L_{1}=Q_{\nu_{1}}$, and $\mathscr{W}=S_{K}, \mathscr{W}_{1}=S_{K_{1}}$.

Lemma 7.2.1. $\forall w \in \mathscr{W} \backslash \mathscr{W}_{1}: \exists w^{\prime} \in \mathscr{W}_{1}: w L^{\circ} \cap L^{\circ} \subset w^{\prime} L_{1}^{\circ}$.

Proof. We will denote the position of $a$ in $\nu$ by $\alpha$. The beginning of $\eta^{(2)}$ in $\eta$ will be at position $\beta^{\prime}$, and $x$ will be at position $\beta$.

First we make a few remarks which follow either from the definitions or else by counting.

(1) $\bigcup_{i<\alpha} L_{i}=\bigcup_{i<\beta^{\prime}} K_{i}$, and the second decomposition is a refinement of the first.

(2) $\bigcup_{i \geq \alpha+1} L_{i}=K_{1, \beta+1}$.

(3) $L_{\alpha}=\bigcup_{\beta^{\prime} \leq i \leq \beta} K_{1, i}$.

(4) $\mathscr{W} \backslash \mathscr{W}_{1}=\prod_{i \neq \beta} S_{K_{i}} \times\left(S_{K_{\beta}} \backslash\left(S_{K_{1, \beta}} \times S_{K_{1, \beta+1}}\right)\right)$.

(5) $L_{1}^{\circ}=L^{\circ} \backslash\left(L_{\alpha} \times L_{\alpha+1}\right)$.

Now we will try to bound the sets $w L^{\circ} \cap L^{\circ}$, where $w \in \mathscr{W} \backslash \mathscr{W}_{1}$. To this end, we compute

$$
\begin{aligned}
L^{\circ} & =\left(\bigcup_{\substack{i<j \\
i<\alpha}} L_{i} \times L_{j}\right) \cup\left(\bigcup_{\substack{i<j \\
i \geq \alpha}} L_{i} \times L_{j}\right) \\
& \subset\left(\bigcup_{\substack{i<j \\
i<\alpha}} L_{i} \times L_{j}\right) \cup\left[\left(\bigcup_{i \geq \alpha} L_{i}\right) \times\left(\bigcup_{j \geq \alpha+1} L_{j}\right)\right] .
\end{aligned}
$$

Using (1), we see that $\bigcup_{j>i, i<\alpha} L_{i} \times L_{j}$, and $\bigcup_{i \geq \alpha} L_{i}$ are $\mathscr{W}$-invariant.

Furthermore, by (2) and (4), $\bigcup_{j \geq \alpha+1} L_{j}$ cannot be $\mathscr{W}$-invariant if $w \in$ $\mathscr{W} \backslash \mathscr{W}_{1}$. Hence, there exists a $p$ in $\bigcup_{j \geq \alpha+1} L_{j}$ such that

$$
L^{\circ} \cap w L^{\circ} \subset\left(\bigcup_{\substack{i<\alpha \\ j>i}} L_{i} \times L_{j}\right) \cup\left[\left(\bigcup_{\substack{i \geq \alpha \\ j>i}} L_{i} \times L_{j}\right) \backslash\left(\bigcup_{i \geq \alpha} L_{i} \times\{p\}\right)\right] .
$$

We will now show that the right-hand side of $(31)$ is contained in some $w^{\prime} L_{1}^{\circ}$ for $w^{\prime} \in \mathscr{W}_{1}$. Assume that $L_{\alpha+1}=\{q\}$. We define $w^{\prime}=(p, p-1, \ldots, q)$. By (2), we see that $w^{\prime} \in \mathscr{W}_{1}$. We will now decompose $L_{1}^{\circ}$ (using (5)).

$$
L_{1}^{\circ}=\left(\bigcup_{\substack{i<\alpha \\ j>i}} L_{i} \times L_{j}\right) \cup\left[\left(\bigcup_{\substack{i=\alpha \\ j>i}} L_{i} \times L_{j}\right) \backslash\left(L_{\alpha} \times\{q\}\right)\right] \cup\left(\bigcup_{\substack{>>\alpha \\ j>i}} L_{i} \times L_{j}\right) .
$$


Here

Therefore,

$$
\bigcup_{\substack{i>\alpha \\ j>i}} L_{i} \times L_{j}=\left\{\left(p_{1}, p_{2}\right) \mid p_{1}, p_{2} \in Q, p_{2}>p_{1} \geq q\right\}
$$

$$
w^{\prime}\left(\bigcup_{\substack{i>\alpha \\ j>i}} L_{i} \times L_{j}\right) \supset\left(\bigcup_{\substack{i>\alpha \\ j>i}} L_{i} \times L_{j}\right) \backslash\left(\bigcup_{i>\alpha} L_{i} \times\{p\}\right) .
$$

(This is the key point.)

Using (2), (3), and (32), we deduce that

$$
\begin{aligned}
w^{\prime} L^{\prime \circ} \supset & \left(\bigcup_{\substack{i<\alpha \\
j>i}} L_{i} \times L_{j}\right) \cup\left[\left(\bigcup_{\substack{i=\alpha \\
j>i}} L_{i} \times L_{j}\right) \backslash\left(L_{\alpha} \times\{p\}\right)\right] \\
& \cup\left[\left(\bigcup_{\substack{i>\alpha \\
j>i}} L_{i} \times L_{j}\right) \backslash\left(\bigcup_{i>\alpha} L_{i} \times\{p\}\right)\right] \\
= & \left(\bigcup_{\substack{i<\alpha \\
j>i}} L_{i} \times L_{j}\right) \cup\left[\left(\bigcup_{\substack{i \geq \alpha \\
j>i}} L_{i} \times L_{j}\right) \backslash\left(\bigcup_{i \geq \alpha} L_{i} \times\{p\}\right)\right],
\end{aligned}
$$

which is precisely the right-hand side of (31).

Corollary 7.2.2. $\left(G\left(\lambda_{\eta_{1}}, X_{\lambda_{\nu_{1}}}\right)\right.$ is a reduction pair for $\left(G\left(\lambda_{\eta}\right), X_{\lambda_{\nu}}\right)$.

Proof. Immediate from Lemmas 7.1.3 and 7.2.1.

7.3. Good pairs of ordered partitions. In this subsection, we will prove that for certain pairs of partitions $(\eta, \nu)$, the pair $\left(G\left(\lambda_{\eta}\right), X_{\lambda_{\nu}}\right)$ is constructible. We will build upon the result obtained in Corollary 7.2.2.

Definition 7.3.1. Let $(\eta, \nu)$ be a pair of ordered partitions of $n$. Then we say that $(\eta, \nu)$ is good if one of the following holds:

(1) $\eta \triangleleft \nu$.

(2) $\eta=\left(\eta^{(1)}, \eta^{(2)}, x\right), \nu=\left(\nu^{(1)}, a, \nu^{(2)}\right)$, where
(a) $\eta^{(1)}, \eta^{(2)}, \nu^{(1)}, \nu^{(2)}$ are ordered partitions.
(b) $x, a \in \mathbf{N}_{0}$.
(c) $\eta^{(1)} \triangleleft \nu^{(1)}$.
(d) $\nu^{(2)}$ consists entirely of ones.
(e) $a+x>n-\sum \nu^{(1)}$.

Lemma 7.3.2. Assume that $\eta=\left(\eta^{(1)}, \eta^{(2)}, x\right)$ and $\nu=\left(\nu^{(1)}\right.$, a) are ordered partitions of $n$ such that $\eta^{(1)} \triangleleft \nu^{(1)}$. Then $\eta \triangleleft \nu$.

Proof. Clear. 
Lemma 7.3.3. Let $\eta=\left(\eta^{(1)}, \eta^{(2)}, x\right)$ and $\nu=\left(\nu^{(1)}, a, \nu^{(2)}\right)$ be ordered partitions of $n$ such that $\eta^{(1)} \triangleleft \nu^{(1)}$ and $\nu^{(2)}$ consists entirely of ones. Assume that $a+x=n-\sum \nu^{(1)}$. Then the pair $(\eta, \nu)$ is good.

Proof. By Lemma 7.3.2, we may assume that $\nu^{(2)}=\left(1, \nu^{(3)}\right)$. We deduce $a+x=n-\sum \nu^{(1)}=n-\sum \eta^{(1)}=\sum \eta^{(2)}+x$ or $\sum \eta^{(2)}=a$. Hence, $\eta^{(3)}=\left(\eta^{(1)}, \eta^{(2)}\right) \triangleleft\left(\nu^{(1)}, a\right)=\nu^{(4)}$. We then rewrite $\eta=\left(\eta^{(3)}, x\right), \nu=$ $\left(\nu^{(4)}, 1, \nu^{(3)}\right)$. Now we see that $(\eta, \nu)$ satisfies the first four conditions of Definition 7.3.1 (2) (making the appropriate translations).

To check condition (2)(e), we observe that $1+x>n-\sum \nu^{(4)}$ since $n-$ $\sum \nu^{(4)}=n-\sum \nu^{(1)}-a=x$.

Assume now that we have a good pair of partitions $(\eta, \nu)$, but not $\eta \triangleleft \nu$. Then $\eta=\left(\eta^{(1)}, \eta^{(2)}, x\right), \nu=\left(\nu^{(1)}, a, \nu^{(2)}\right)$ as in Definition 7.3.1. Furthermore, by Lemma 7.3.2, $\nu^{(2)}$ is nonempty and hence $\nu^{(2)}=\left(1, \nu^{(3)}\right)$. We can then define $\left(\eta_{1}, \nu_{1}\right)$ as in 7.2, i.e., $\eta_{1}=\left(\eta^{(1)}, \eta^{(2)}, x-n+\sum \nu^{(1)}+a, n-\right.$ $\left.\sum \nu^{(1)}-a\right)$ and $\nu_{1}=\left(\nu^{(1)}, a+1, \nu^{(2)}\right)$.

Lemma 7.3.4. $\left(\eta, \nu_{1}\right),\left(\eta_{1}, \nu\right)$, and $\left(\eta_{1}, \nu_{1}\right)$ are good.

Proof. (1) $\left(\eta, \nu_{1}\right)$ is good because $a+1+x>n-\sum \nu^{(1)}$ (using Definition 7.3.1).

(2) $\left(\eta_{1}, \nu\right)$ is good because $n-\sum \nu^{(1)}-a+a=n-\sum \nu^{(1)}$. Hence, we can apply Lemma 7.3.3.

(3) $\left(\eta_{1}, \nu_{1}\right)$ is good because $n-\sum \nu^{(1)}-a+a+1>n-\sum \nu^{(1)}$.

Corollary 7.3.5. If $(\eta, \nu)$ is a good pair of ordered partitions of $n$, then $\left(G\left(\lambda_{\eta}\right)\right.$, $\left.X_{\lambda_{\nu}}\right)$ is constructible.

Proof. Clear from Corollary 7.2.2, Lemma 7.3.4, and the fact that eventually (1) of Definition 7.3.1 must become true.

Theorem 7.3.6. $\mathbf{T}_{m, n}$ is Cohen-Macaulay for all $(m, n)$.

Proof. As we saw before (eq. 3), $\mathbf{T}_{m, n}=(\operatorname{End}(V) \otimes k[W])^{G}$. The case $n=1$ is trivial. Furthermore, it is easily verified that $\mathbf{T}_{1, n}$ is a free module over its center which is a polynomial ring. Hence, the result is clear. $\mathbf{T}_{2,2}$ was treated in [5], [18].

Hence, it remains to consider the cases $(m, n) \geq(2,3)$ and $\geq(3,2)$.

It is well known that $\operatorname{End}(V)=k \oplus \operatorname{End}(V)^{0}$, where the elements of $\operatorname{End}(V)^{0}$ are those endomorphisms of $V$ having trace 0 . This is an irreducible $G$ representation. Since $k[W]^{G}$ is Cohen-Macaulay by the Hochster-Roberts Theorem, it suffices to look at the case $U=\operatorname{End}(V)^{0}$. It is easy to verify that the character of $U^{*}$ is strongly critical for $(G, W)$ if $(m, n) \geq(2,3)$ or $\geq(3,2)$. Furthermore, if $m \geq 2$, then the action on $X$ is generically free, and hence $X$ has a stable point by Theorem 6.1.8. Hence, the only thing that has to be proved, to apply Theorem 6.1.9, is that $X^{u}$ is constructible. However, by Lemma 7.1.2, $X^{u}=G\left(\lambda_{\eta_{\max }}\right) X_{\lambda_{\eta_{\min }}}$, and according to Definition 7.3.1, $\left(\eta_{\max }, \eta_{\min }\right)$ is a good 
pair of ordered partitions $\left(\eta^{(1)}, \eta^{(2)}\right.$, and $\nu^{(1)}$ are empty in this case). Hence, we may apply Corollary 7.3.5.

Remark 7.3.7. It may be somewhat surprising that the cases $m=1$ and $(m, n)$ $=(2,2)$ play a special role in the above argument. However, it is easily verified that

$$
P\left(\mathbf{T}_{1, n}, t\right)=\frac{1}{(1-t)^{2}\left(1-t^{2}\right) \cdots\left(1-t^{n-1}\right)}
$$

and

$$
P\left(\mathbf{T}_{2,2}, t\right)=\frac{1}{(1-t)^{4}\left(1-t^{2}\right)} .
$$

Hence, they satisfy the functional equations

$$
P\left(\mathbf{T}_{1, n}, 1 / t\right)=(-1)^{n} t^{\left(n^{2}-n+2\right) / 2} P\left(\mathbf{T}_{1, n}, t\right)
$$

and

$$
P\left(\mathbf{T}_{2,2}, 1 / t\right)=-t^{6} P\left(\mathbf{T}_{2,2}, t\right)
$$

which are different from the functional equation for $(m, n) \geq(2,3)$ or $\geq$ $(3,2)$ (as predicted by (eq. 4)),

$$
P\left(\mathbf{T}_{m, n}, 1 / t\right)=(-1)^{(m-1) n^{2}+1} t^{m n^{2}} P\left(\mathbf{T}_{m, n}, t\right) \text {. }
$$

For other proofs of this functional equation, we refer to [4], [10], [21]. However, these authors seem to have been unaware of the general result in [20].

\section{REFERENCES}

1. M. Artin, On Azumaya algebras and finite dimensional representations of rings, J. Algebra 11 (1969), 532-563.

2. M. Artin and W. Schelter, Integral ring homomorphisms, Adv. in Math. 39 (1981), 289-329.

3. R. Bott, Homogeneous vector bundles, Ann. of Math. (2) 65 (1957), 203-248.

4. E. Formanek, Functional equations for character series associated with $n \times n$ matrices, Trans. Amer. Math. Soc. 294 (1986), 647-663.

5. E. Formanek, P. Halpin, and W. Li, The Poincaré series of 2 by 2 matrices, J. Algebra 69 (1981), 105-112.

6. R. Hartshorne, Residues and duality, Springer-Verlag, New York, 1966.

7. W. H. Hesselink, Desingularisations of varieties of null forms, Invent. Math. 55 (1979), 141163.

8. M. Hochster and J. Roberts, Rings of invariants of reductive groups acting on regular rings are Cohen-Macaulay, Adv. in Math. 13 (1974), 313-373.

9. F. C. Kirwan, Cohomology of quotients in symplectic and algebraic geometry, Math. Notes, no. 31, Princeton Univ. Press, Princeton, NJ, 1984.

10. L. le Bruyn, Trace rings of generic $2 \times 2$ matrices, Mem. Amer. Math. Soc., no. 363, Amer. Math. Soc., Providence, RI, 1987.

11. L. LeBruyn and C. Procesi, Etale local structure of matrix invariants and concomittants, Lecture Notes in Math., no. 1271, Springer-Verlag, Berlin and New York, 1986, pp. 143-176.

12. L. LeBruyn and M. Van den Bergh, Regularity of trace rings of generic matrices, J. Algebra 117 (1988), 19-29.

13. G. Kempf, Collapsing of homogeneous bundles, Invent. Math. 37 (1976), 229-239. 
14. D. Mumford, Geometric invariant theory, Springer-Verlag, New York, 1982.

15. V. L. Popov, Stability criteria for the actions of a semisimple group on a factorial manifold, Izv. Akad. Nauk SSSR Ser. Math. 4 (1970), 527-535.

16. C. Procesi, Invariant theory of $n \times n$-matrices, Adv. in Math. 19 (1976), 306-381.

17. __. Trace identities and standard diagrams, Proc. 1978 Conf. on Ring Theory (F. Van Oystaeyen, ed.), Marcel Dekker, New York, 1979, pp. 191-218.

18. L. Small and T. Stafford, Homological properties of generic matrix rings, Israel J. Math. $\mathbf{5 1}$ (1985), 27-32.

19. T. A. Springer, Linear algebraic groups, Progr. Math., Vol. 9, Birkhäuser, Boston, 1981.

20. R. Stanley, Combinatorics and invariant theory, Proc. Sympos. Pure Math., Vol. 34, Amer. Math. Soc., Providence, RI, 1979, pp. 345-355.

21. Y. Teranishi, The Hilbert series of rings of matrix concomittants, Nagoya Math. J. 111 (1988), 143-156.

22. M. Van den Bergh, Cohen-Macaulayness of modules of invariants for $\mathrm{SL}_{2}$ (to appear).

ABSTRACT. In this paper, we prove that trace rings of generic matrics are CohenMacaulay (Theorem 7.3.6). This is done by relating this problem to a conjecture of Stanley about modules of invariants under a reductive group.

We prove a slightly weakened version (Conjecture $3.4^{\prime}$ ) of this conjecture in special cases (Theorem 6.1.8). In particular, we obtain that Conjecture $3.4^{\prime}$ is true for $S L_{2}$ (Remark 6.1.10).

DepartMent of Mathematics, Universiteitsplein 1, UNiversitaire INSTElling ANTWERPEN, 2160 WILRIJK, BELGIUM 